\title{
The impact of the COVID-19 pandemic on international reconstructive collaborations in Africa
}

\author{
Calum S. Honeyman ${ }^{1}$ (D) Vinod Patel ${ }^{2} \cdot$ Abdelwakeel Bakhiet $^{1} \cdot$ Daniel R. Bradley $^{3} \cdot$ Fernando Almas $^{4}$. \\ Dominique Martin ${ }^{5} \cdot$ Mark McGurk $^{6}$
}

Received: 1 July 2021 / Accepted: 17 September 2021 / Published online: 5 October 2021

(c) The Author(s), under exclusive licence to Springer-Verlag GmbH Germany, part of Springer Nature 2021

\begin{abstract}
Background The SARS-CoV-2 (COVID-19) pandemic has catalysed a widespread humanitarian crisis in many low- and middle-income countries around the world, with many African nations significantly impacted. The aim of this study was to quantify the impact of the COVID-19 pandemic on the planning and provision of international reconstructive collaborations in Africa.

Methods An anonymous, 14-question, multiple choice questionnaire was sent to 27 non-governmental organisations who regularly perform reconstructive surgery in Africa. The survey was open to responses for four weeks, closing on the $7^{\text {th }}$ of March 2021. A single reminder was sent out at 2 weeks. The survey covered four key domains: (1) NGO demographics; (2) the impact of COVID-19 on patient follow-up; (3) barriers to the safe provision of international surgical collaborations during COVID-19; (4) the impact of COVID-19 on NGO funding.

Results A total of ten reconstructive NGOs completed the survey (response rate, 37\%). Ethiopia $(n=5)$ and Tanzania $(n=4)$ were the countries where most collaborations took place. Plastic, reconstructive and burns surgery was the most common sub-speciality $(n=7)$. For NGOs that did not have a year-round presence in country $(n=8)$, only one NGO was able to perform reconstructive surgery in Africa during the pandemic. The most common barrier identified was travel restrictions (within country, $n=8$ or country entry-exit, $n=7$ ). Pre-pandemic, 1547 to $\geq 1800$ patients received reconstructive surgery on international surgical collaborations. After the outbreak, $70 \%$ of NGOs surveyed had treated no patients, with approximately 1405 to $\geq 1640$ patients left untreated over the last year.

Conclusions The COVID-19 pandemic has placed huge pressures on health services and their delivery across the globe. This theme has extended into international surgical collaborations leading to increased unmet surgical needs in low- and middle-income countries.

Level of evidence: Not gradable.
\end{abstract}

Keywords International surgical collaborations · Global surgery · Reconstruction · Africa · COVID-19 · SARS-CoV-2

Calum S. Honeyman

c.s.honeyman@gmail.com

1 Department of Plastic, Reconstructive and Burns Surgery, Ninewells Hospital, James Arrott Drive, Dundee DD2 1SG, Scotland, UK

2 Oral Surgery Department, Guys Dental Institute, Guys \& St Thomas NHS Foundation Trust, London, England, UK

3 Department of Head and Neck Surgery, The Royal Marsden Hospital, London, UK
4 Department of Cranio-MaxilloFacial and Reconstructive Surgery, Saint Judes General Hospital, Federal Hospital of Rio de Janeiro, Rio de Janeiro, Brazil

5 Marseille, France

6 Department of Head and Neck Surgery, University College London Hospital, London, England, UK 


\section{Introduction}

The SARS-CoV-2 (COVID-19) pandemic has led to a widespread humanitarian crisis in most low- and middleincome countries (LMIC) around the world [1-3]. Many African countries, with fragile healthcare systems before the outbreak of COVID-19, have been amongst the worst affected. According to the Africa Centres for Disease Control and Prevention, on the $23^{\text {rd }}$ of August 2021, 7.6 million cases of COVID-19 had been recorded on the continent, with an ongoing case mortality rate of $2.6 \%$ (compared to a worldwide average of 2.2\%) [4]. Currently, a widespread lack of basic resources including oxygen, personal protective equipment (PPE), and a vaccination rate of only $2 \%$ across the continent, is perpetuating the impact of COVID-19 in Africa [5].

Pre-pandemic, many non-governmental organisations (NGOs) routinely facilitated life-changing reconstructive surgery in Africa, using a collaborative international model to deliver patient care. Previously referred to as short-term reconstructive missions, these collaborations involved a partnership between visiting and local surgical teams $[1,6]$. The aim of this study was to quantify the impact of the COVID-19 pandemic on the planning and provision of international reconstructive collaborations in Africa.

\section{Methods}

An anonymous, 14-question, multiple choice, online survey was designed using Google Forms (Supplementary Data 1). The survey was emailed to named contacts for 27 non-governmental organisations (NGO) who regularly facilitate reconstructive surgery in Africa using an international, collaborative model of surgical care delivery. No incentives were offered to complete the survey. NGOs were identified using a comprehensive google search on the $18^{\text {th }}$ of July 2021 . The final results of the search were cross referenced with NGO staff currently working in the field to ensure as many relevant NGOs as possible were included. The survey was open to responses for four weeks (07/02/21-07/03/21). A single reminder was sent out at 2 weeks. The survey covered four key domains: (1) NGO demographics; (2) the impact of COVID-19 on patient follow-up; (3) barriers to the safe provision of international surgical collaborations during COVID-19; (4) the impact of COVID-19 on NGO funding. Data was analysed using Graph Pad Prism v9. Parametric data is presented as mean and standard deviation (SD) and non-parametric data as median and interquartile range (IQR).

\section{Results}

A total of 10/27 (37\%) reconstructive NGOs responded to the survey. Countries in which international surgical collaborations operated are summarised in Table 1. Ethiopia $(n=5)$ and Tanzania $(n=4)$ were the most frequent locations. The most common sub-specialty was plastic, reconstructive and burns surgery $(n=7)$, followed by craniofacial and oral and maxillofacial surgery (Fig. 1). Two NGOs surveyed perform reconstructive surgery all year round with a continuous presence in their country of operation, and were therefore able to continue in some capacity after the outbreak of COVID-19. For the eight NGOs that did not have a year-round presence, the mean number of international collaborations performed per NGO per year before the outbreak of COVID-19 was three (SD 1, range 1-5). Post-pandemic, only one NGO successfully performed any reconstructive surgery on an international surgical collaboration that employed visiting surgical teams from outside Africa.

Prior to the COVID-19 pandemic, the total number of patients undergoing reconstructive surgery on international surgical collaborations was between 1547 and $\geq 1800$ (range per collaborations 11-20 to $>100$ ). Figure 2 shows the impact of COVID-19 on the number of patients undergoing reconstructive surgery, per international surgical collaboration, before and after the start of the pandemic (March 2020). After the outbreak, 70\% of NGOs were unable to treat any patients. In this representative sample of ten NGOs surveyed, between 1405 and $\geq 1640$ patients have been left untreated over the last year. For NGOs able to perform some reconstructive surgery, between 142 and $\geq 160$ patients have been treated. Hence, COVID-19 disruption has led to a treatment
Table 1 African country or countries international reconstructive collaborations operate in

\begin{tabular}{ll}
\hline Country & $\mathrm{n}$ \\
\hline Ethiopia & 5 \\
Tanzania & 4 \\
Ghana & 2 \\
Liberia & 2 \\
Uganda & 2 \\
South Africa & 2 \\
The Democratic Republic of & 2 \\
the Congo & \\
Nigeria & 2 \\
Cote d'Ivoire & 1 \\
Zambia & 1 \\
Kenya & 1 \\
Egypt & 1 \\
Libya & 1 \\
Sierra Leone & 1 \\
\hline
\end{tabular}




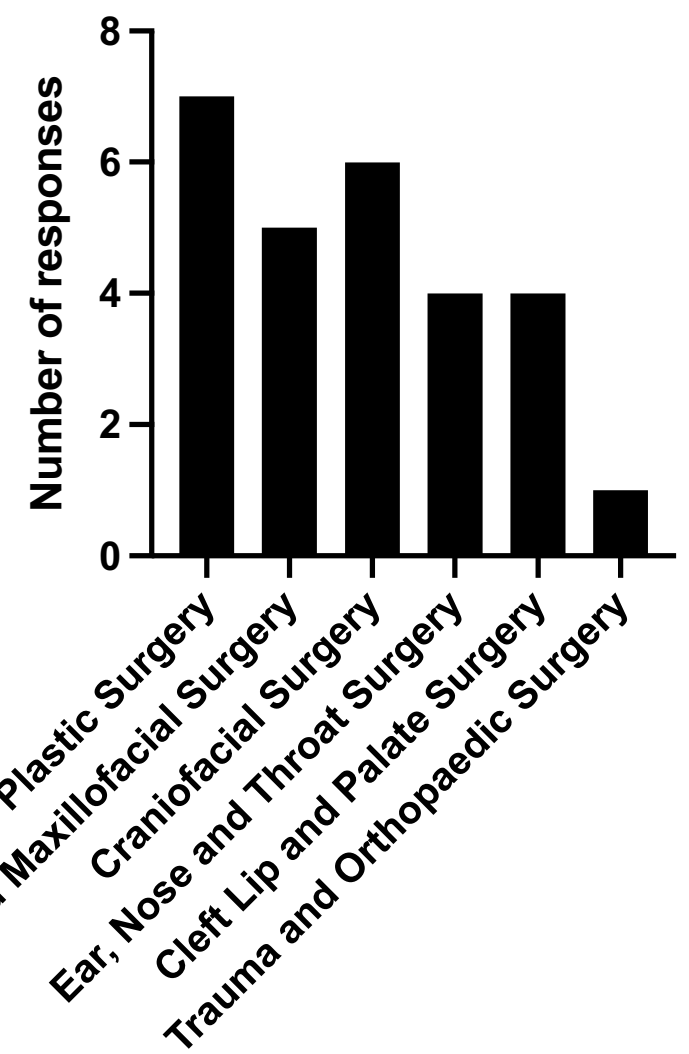

\section{Sub-specialty}

Fig. 1 Reconstructive sub-specialty performed on international reconstructive collaborations in Africa

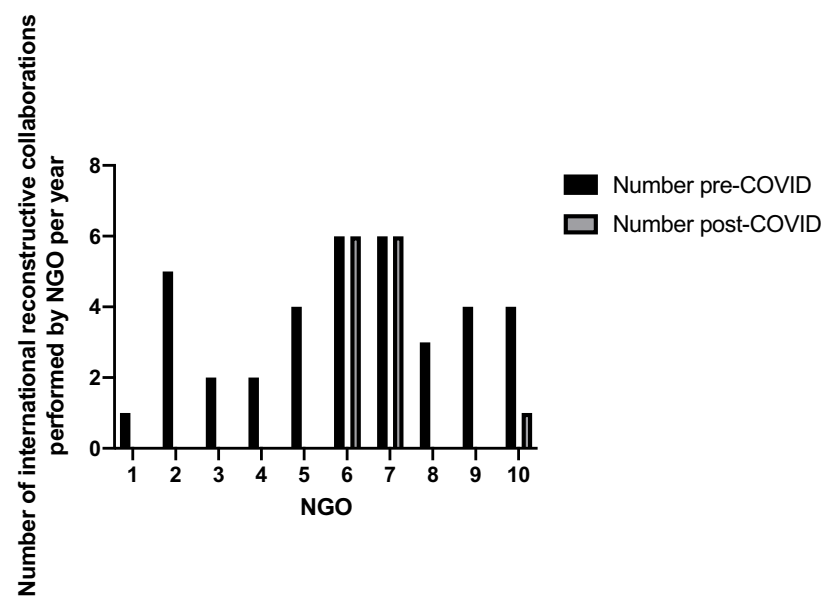

Fig. 2 Number of international reconstructive collaborations performed per NGO per year in Africa

reduction of approximately $90 \%$ since March 2020. Significant barriers are summarised in Fig. 3, with travel restriction within country as well as entry and exiting most commonly cited as the main difficulty.
The contrast in follow-up methods employed before and after the outbreak of the COVID-19 pandemic can be seen in Fig. 3. Prior to the outbreak of COVID-19, 70\% of NGOs performed some form of in-person follow-up. The number of NGOs that were unable to perform any follow-up (face-face or remote follow-up) increased from two pre-COVID-19 to five during the pandemic. Figure 4 summarises key barriers to following up patients before the COVID-19 outbreak, including significant distances for patients to travel to return for follow-up $(n=8)$, financial limitations experienced by patients to return $(n=7)$ and an inability to contact patients after discharge due to no mobile phone coverage $(n=4)$. Figure 5 demonstrates significant barriers facing NGOs after COVID-19 outbreak, and Fig. 6 presents changes to the timing of patient follow-up before and after the outbreak of COVID-19. Seventy percent of responses were 'other', reflecting the absence of international reconstructive collaborations currently being performed.

Sixty percent of the NGOs surveyed strongly agree $(n=4)$ or agree $(n=2)$ with the statement that "the COVID-19 pandemic has had a significant negative impact on charity finances", with the remaining neutral $(n=3)$ or in disagreement $(n=1)$. Seventy percent of NGOs strongly agree $(n=3)$ or agree $(n=4)$ with the statement that "the COVID19 pandemic has had a significant negative impact on the ability of the charity fundraise", with the remaining neutral $(n=2)$ or in disagreement $(n=1)$. Six NGOs surveyed had seen a shift in financial support from international domestic programmes. In contrast, six NGOs had seen major donors remove restrictions on the allowed uses of funding, giving more flexibility.

Of the eight NGOs surveyed that do not have a year-round presence, the mean predicted time to restart international surgical collaborations was 3 months (range 1-6 months). For both NGOs with a continuous presence, the next reconstructive surgery is planned to be carried out within the next 6 months.

\section{Discussion}

This study highlights the significant logistical and financial barriers preventing reconstructive NGOs from carrying out thousands of life-changing operations in Africa since the outbreak of COVID-19 in March 2020.

This representative survey of ten such NGOs clearly highlights the ever-increasing burden of untreated reconstructive surgical pathology in Africa, directly related to barriers created by the COVID-19 pandemic. Seventy percent of NGOs have been unable to conduct any international surgical collaborations since March 2020, which has led to between 1547 and $\geq 1800$ patients, across all reconstructive sub-specialties, left untreated. As this survey was limited by 
Fig. 3 Method of patient followup before and after COVID-19 outbreak
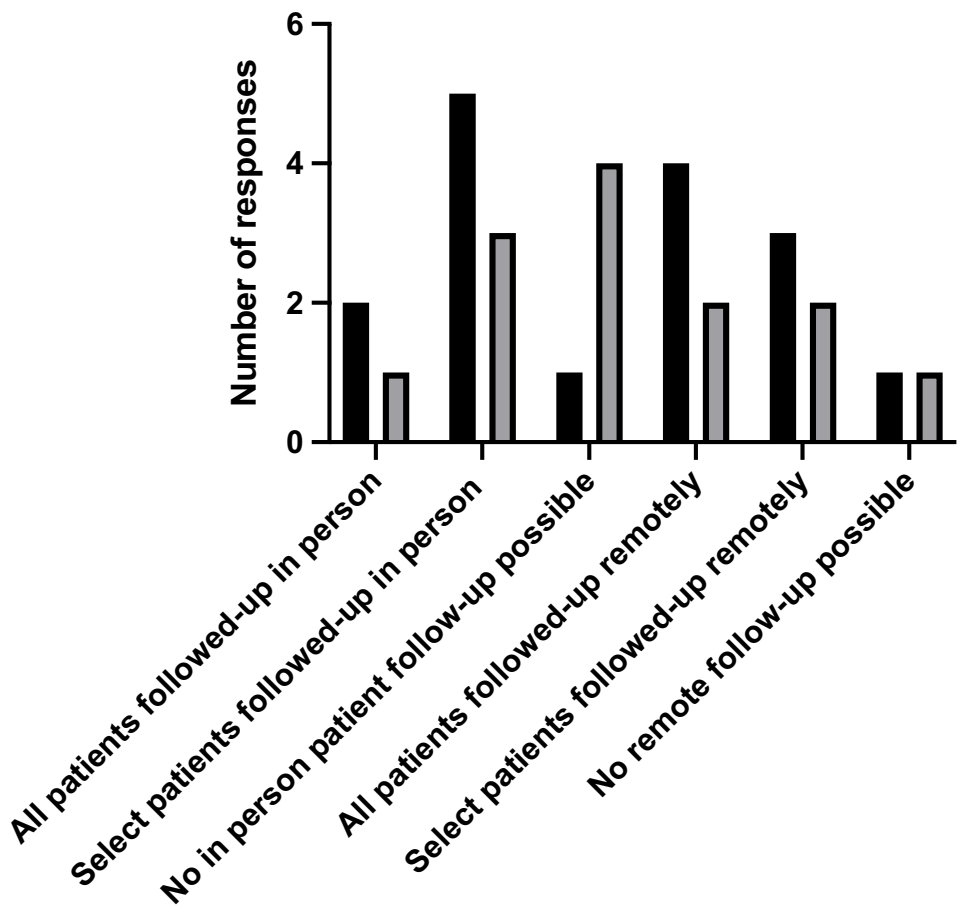

Method of patient follow-up

a response rate of $37 \%$, the true number of untreated patients is likely to be far higher.

Although the mean predicted time to restart reconstructive collaborations for NGOs surveyed was 3 months, the third wave of COVID-19 infections currently being experienced by many European countries could be replicated in other countries around the world, and is particularly concerning for many African countries with only skeleton vaccination programmes running at present [7]. This may heighten the barriers cited by many NGOs in this study, including continued international travel restrictions, disruption to internal travel within African countries, further depletion of PPE supplies, and significant NGO financial strain, particularly for smaller charities with little reserve. The multitude of logistical barriers detailed in this study threatens the safe resumption of international surgical collaborations within the next 3 to 6 months, and may increasingly impact those with a continuous year-round presence, who until now have been able to perform some reconstructive surgery.

Despite encouraging international vaccine support programmes like Covax, a WHO-backed initiative aiming to supply 600 million doses to Africa by the end of 2021, progress in many LMICs will be slow, lagging far behind that of higher income countries [8]. According to the 'ourworldindata' website, as of the $13^{\text {th }}$ of March 2021, only 0.5 vaccine doses per 100,000 people had been given in Africa, compared to 37 doses per 100,000 people in the United Kingdom [9]. Furthermore, within Africa, vaccination programmes may differ greatly between countries. Tanzania, for example, the second most prevalent country where NGOs in the survey work, has no plan to acquire vaccines at the present time [8].

The lengthy delays to treatment will inadvertently lead to advancement of pathology, which for some will render
Fig. 4 Barriers to patient follow-up before the COVID-19 outbreak

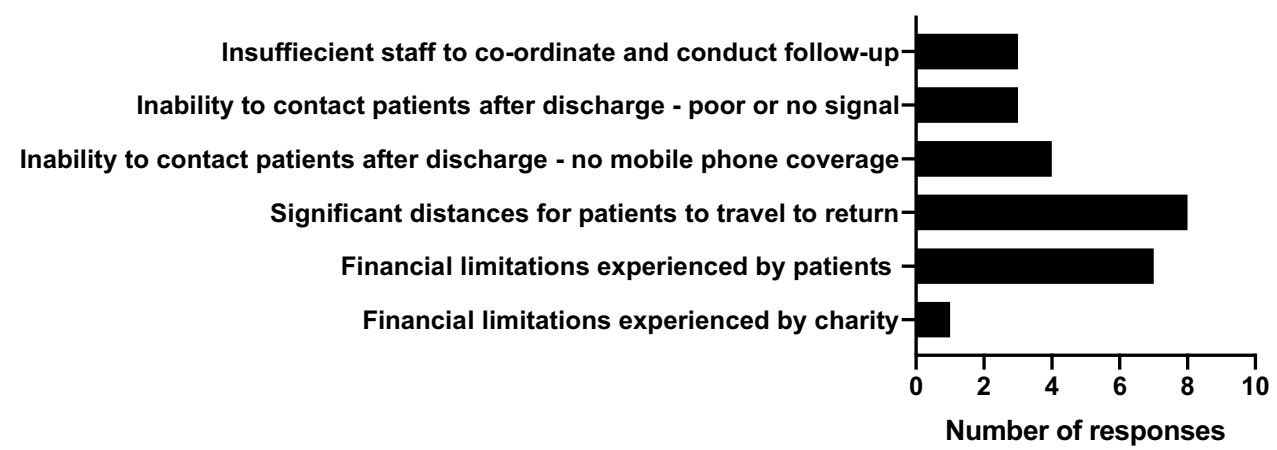




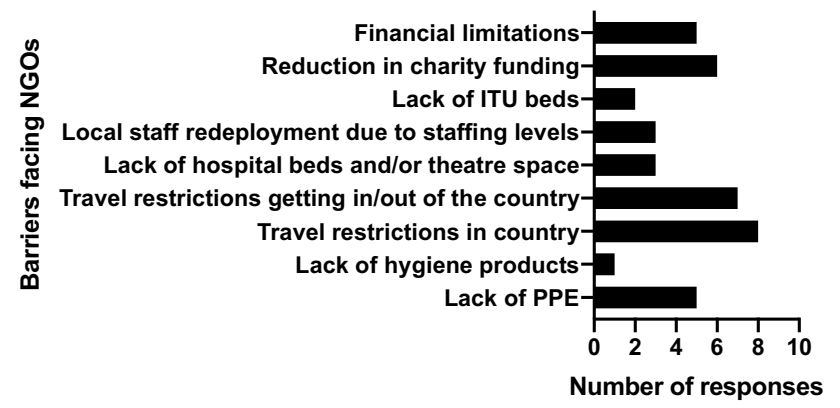

Fig. 5 Significant barriers facing NGOs after COVID-19 outbreak

their condition inoperable, and for many will lead to a spiral of worsening pre-operative nutritional status, perpetuated by worsening poverty. Finally, Ethiopia, which is the country where most reconstructive surgery takes place, is in the midst of a devastating civil war, with almost 15,000 deaths since January 1, 2021, making international reconstructive surgery highly unlikely for the foreseeable future [10]. The combination of war and COVID-19 will likely perpetuate the cycle of poverty and malnutrition that leads to increased prevalence of conditions like Noma (Cancrum Oris), a disfiguring orofacial gangrene with significant mortality, therefore increasing the burden of facial reconstructive pathology in Ethiopia over the coming years [11].

Prior to the outbreak of COVID-19, follow-up after patient discharge from international surgical collaborations in Africa was challenging [12]. This study reiterates many of the traditionally cited barriers to achieving consistent patient follow-up, including financial limitations for patients, significant distances that patients are required to travel and an inability to contact patients after they are discharged due to lack of mobile phones coverage. Although many NGOs are not currently running international reconstructive collaborations in Africa, and as such new patients will not require follow-up, it is clear that follow-up for patients operated on before March 2020 will have been additionally impacted by

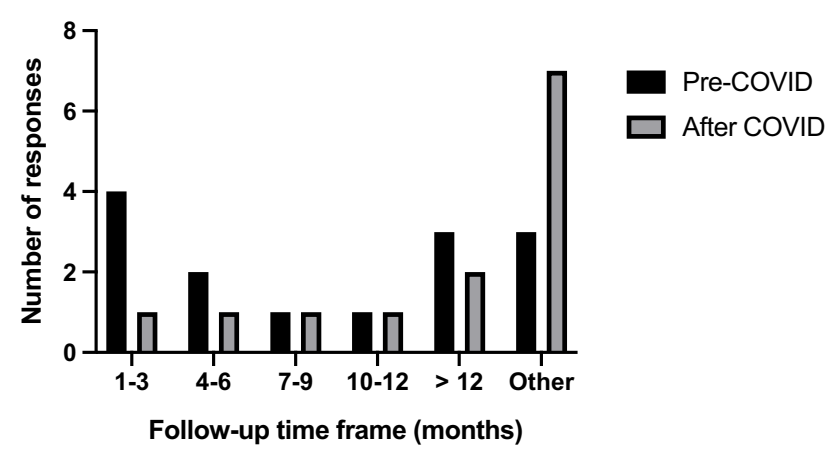

Fig. 6 Patient follow-up time frame before and after COVID-19 outbreak logistical issues due to COVID-19. It is encouraging that many of the NGOs surveyed had excellent pre-pandemic follow-up protocols, often following up major cases in person and others remotely. Now more than ever, exploring and refining methods for remote follow-up is essential, and may lead to positive long-term changes that are more time and cost-effective going forward [13].

Survey responses identified significant financial pressures, including a reduction in fundraising opportunities, faced by $70 \%$ of the NGOs surveyed. Six NGOs reported a shift from international to domestic support programmes. In contrast, six NGOs have seen major donors remove restrictions on funds received, leading to greater flexibility (e.g. to purchase PPE) and cost-free extensions, as well as the ability to renegotiate specific project funding. One NGO reported a promising experience using social media as a new avenue for fundraising, allowing them to provide support for partner hospitals facing closure due to COVID-19.

The pandemic has forced some NGOs to rethink their model, exploring new collaborations and ways of supporting in-country care. It is worth noting that the only two NGOs who continued to perform reconstructive surgery over the last year, albeit in a reduced capacity, have been those with a sustainable, year-round presence in the country in which they operate. For many international collaborations that have been running for many years, the opportunity to support a transition to surgical care delivered by local surgeons, with additional funding support, could be considered [6]. In the short-term, some NGOs have switched to fundraise for PPE, hygiene products and basic medical equipment [1]. Additional support will also be required to support nutritional programmes, particularly for cleft lip and palate and in advance of major reconstructive surgery when international collaborations do begin again. Many NGOs have managed to harness online educational events (e.g. BAPRAS, BFIRST, BBA webinars) to stay connected, even if in-person courses and hands-on training cannot go ahead [14].

\section{Study limitations}

This study has some relevant limitations. Firstly, the response rate of this survey response rate was 37\% (10/27 NGOs invited); therefore, our results only represent a portion of the greater number of NGOs conducting reconstructive surgery in Africa. However, we feel our results are widely applicable as they cover a wide spectrum of African countries, sub-specialties, charity sizes and funding structures. A response rate of greater than $20 \%$ is often considered the baseline for publication of survey-based research, but absolute numbers in this study are low as this is a relatively niche topic within global surgery [15]. In addition, although a comprehensive google search was performed to identify relevant NGOs conducting reconstructive surgery in Africa, 
it is possible some may have been inadvertently missed out, despite cross checking with NGO administration staff working in the field.

\section{Conclusions}

This survey clearly highlights the evolving humanitarian crisis facing many African countries due to the COVID-19 pandemic, with a corresponding unmet reconstructive need set to worsen over the coming months and years. There is a clear need for additional support for NGOs facilitating reconstructive surgery. To bridge the gap, attention needs to be directed towards basic aid, vaccination programmes, remote follow-up, ongoing online educational events and a significant nutritional drive before the next iteration of surgeries are performed.

Supplementary Information The online version contains supplementary material available at https://doi.org/10.1007/s00238-021-01892-4.

Acknowledgements We would like to thank all of the reconstructive NGOs that contributed data to this survey, and wish them all the best in the challenging months ahead

\section{Declarations}

Ethical approval All data provided was pooled. All procedures performed in studies involving human participants were in accordance with the ethical standards of the institutional and/or national research committee and with the 1964 Helsinki Declaration and its later amendments or comparable ethical standards. No ethics approval was required in this survey-based study.

Consent to participate Surveyed organisations provided inherent informed consent to have anonymised data they submitted used for peer-review publication, prior to their voluntary participation in this study.

Conflict of interest Calum Honeyman, Vinod Patel, Abdelwakeel Bakhiet, Daniel R Bradley, Fernando Almas, Dominique Martin and Mark McGurk declare no competing interests.

\section{References}

1. Honeyman C, Patel V, Almas F, Bradley D, Martin D, McGurk M (2020) Short-term surgical missions to resource-limited settings in the wake of the COVID-19 pandemic. J Plast Reconstr Aesthet Surg. Churchill Livingstone. https://doi.org/10.1016/j.bjps.2020. 08.048.

2. Fuertes V, Monclús E, Agulló A (2020) Current impact of Covid-19 pandemic on Spanish plastic surgery departments: a multi-center report. Eur J Plast Surg 43(4):483-490. https://doi. org/10.1007/S00238-020-01686-0

3. Khashaba H, Ng L, Osmani O, Chalmers R (2020) COVID-19 and plastic surgery: a UK plastic surgery unit experience. Eur J Plast Surg 43(6): 1. https://doi.org/10.1007/S00238-020-01703-2

4. "Coronavirus Disease 2019 (COVID-19) - Africa CDC." [Online]. Available: https://africacdc.org/covid-19/. [Accessed: 24 Aug 2021].

5. "Covid-19 Africa: What is happening with vaccine supplies? BBC News." [Online]. Available: https://www.bbc.co.uk/news/ 56100076. [Accessed: 24 Aug 2021].

6. Jemec B et al (2021) The governance of overseas surgical collaborations - BFIRST/BSSH. J Plast Reconstr Aesthetic Surg 74(2):396-400. https://doi.org/10.1016/j.bjps.2020.08.022

7. Third Covid wave sweeps across EU and forces new restrictions | Coronavirus | The Guardian. The Gaurdian, 14-Mar-2021. [Online]. Available: https://www.theguardian.com/world/2021/ mar/14/third-covid-wave-sweeps-across-eu-and-forces-new-restr ictions. [Accessed: 15 Mar 2021]

8. Covid-19 Africa: Who is getting the vaccine? - BBC News, $B B C$ News, 20-Feb-2021. [Online]. Available: https://www.bbc.co.uk/ news/56100076. [Accessed: 15 Mar 2021]

9. Coronavirus (COVID-19) Vaccinations - Statistics and Research - Our World in Data, Ourworldindata, 13-Mar-2021. [Online]. Available: https://ourworldindata.org/covid-vaccinations?count ry $=\sim$ ETH. [Accessed: 15 Mar 2021].

10. EEPA HORN No. 103 - 15 March 2021, Situation Report EEPA HORN No. 103 - 15 March 2021, 15-Mar-2021. [Online]. Available: https://www.eepa.be//wp-content/uploads/2020/11/Situa tion-Report-EEPA-Horn-No.-103-15-March-2021.pdf. [Accessed: 15 Mar 2021].

11. Srour ML, Marck K, Baratti-Mayer D (2017) Noma: overview of a neglected disease and human rights violation. Am J Trop Med Hyg 96(2):268-274. https://doi.org/10.4269/ajtmh.16-0718

12. Honeyman $\mathrm{C}$ et al (2020) Long-term outcomes associated with short-term surgical missions treating complex head and neck disfigurement in Ethiopia: a retrospective cohort study. J Plast Reconstr Aesthetic Surg. https://doi.org/10.1016/j.bjps.2019.12. 009

13. Bradley D et al (2020) Smartphones can be used for patient follow-up after a surgical mission treating complex head and neck disfigurement in Ethiopia: results from a prospective pilot study. J Plast Reconstr Aesthetic Surg. https://doi.org/10.1016/j.bjps.2020. 10.025

14. Ali SR, Dobbs TD, Whitaker IS (2020) Webinars in plastic and reconstructive surgery training - a review of the current landscape during the COVID-19 pandemic. J Plast Reconstr Aesthetic Surg. 1-2. https://doi.org/10.1016/j.bjps.2020.05.038

15 Johnson TP, Wislar JS (2012) Response rates and nonresponse errors in surveys. JAMA - J Am Med Assoc 307(17):1805-1806. https://doi.org/10.1001/jama.2012.3532

Publisher's Note Springer Nature remains neutral with regard to jurisdictional claims in published maps and institutional affiliations. 